\title{
One-Dimensional Octacyanomolybdate-Based Cu(II)-Mo(V) Bimetallic Assembly with a Novel Rope-Ladder Chain Structure
}

\author{
Young Sin You, ${ }^{\dagger}$ Dohyeon Kim, ${ }^{\ddagger}$ Youngkyu Do, ${ }^{\ddagger}$ Sang Jun Oh, ${ }^{\S}$ and Chang Seop \\ Hong*, ${ }^{\dagger}$
}

Department of Chemistry and Center for Electro- and Photo-Responsive Molecules, Korea University, Seoul 136-701, Korea; Department of Chemistry, School of Molecular Science BK-21 and Center for Molecular Design and Synthesis, KAIST, Daejon 305-701, Korea; and Korea Basic Science Institute, Daejeon 305-333, Korea.

\section{Supporting Information:}

The exact expression used to reproduce the susceptibility of the pentameric unit $\left(\chi_{\mathrm{p}}\right)$ is indicated as follows:

$$
\chi_{\mathrm{p}}=0.12505 \mathrm{~g}^{2} / \mathrm{T}(\mathrm{A} / \mathrm{B})
$$

where

$$
\begin{aligned}
& A=1.5 \exp (0.720779 p)+15 \exp (1.118869 p)+1.5 \exp (1.267068 p)+15 \exp (1.618869 p) \\
& +1.5 \exp (2.134993 \mathrm{p})+15 \exp (2.236903 \mathrm{p})+1.5 \exp (2.516591 \mathrm{p})+15 \exp (2.736903 \mathrm{p}) \\
& +52.5 \exp (2.927886 \mathrm{p}) \\
& \mathrm{B}=2+2 \exp (0.720779 p)+4 \exp (1.118869 p)+2 \exp (1.267068 p)+4 \exp (1.618869 p)+ \\
& 2 \exp (2.134993 p)+4 \exp (2.236903 p)+2 \exp (2.516591 p)+4 \exp (2.736903 p)+ \\
& 6 \exp (2.927886 \mathrm{p}) \text {, } \\
& \text { and } \mathrm{p}=\mathrm{J}_{\mathrm{p}} / \mathrm{kT} \text {. }
\end{aligned}
$$

